\title{
Diversité piscicole et paramètres démographiques des principales espèces de poisson du réservoir de Samandeni, Burkina Faso
}

Mahamoudou Minoungou , Raymond Ouedraogo \& Adama Oueda

\begin{abstract}
Mahamoudou Minoungou : étudiant en thèse, Burkinabè. 01 BP 7021 Ouagadougou 01, Université Joseph Ki Zerbo, UFR/SVT, Département de Biologie et Physiologie Animales, Laboratoire de Biologie et Écologie Animales - Ouagadougou - Burkina Faso. Auteur correspondant : minoungou_mahamadou@yahoo.fr +226 70312800
\end{abstract}

Raymond Ouedraogo : PhD, Burkinabè. Département Environnement et Forêts, Institut de l'Environnement et de Recherches Agricoles, 04 BP 8645, Ouagadougou. ouedray@yahoo.com +226 71801642/78338408/75886600

Adama Oueda : PhD, Enseignant-chercheur, Burkinabè. 01 BP 7021 Ouagadougou 01, Université Joseph Ki Zerbo, UFR/SVT, Département de Biologie et Physiologie Animales, Laboratoire de Biologie et Écologie Animales - Ouagadougou - Burkina Faso. oueda14@yahoo.fr +226 71585930

DOI: $10.25518 / 2295-8010.1948$

\section{Résumé :}

La gestion de la pêcherie du réservoir de Samandeni au Burkina Faso, nouvellement mise en eau en 2017 nécessite des informations fiables qui sont pour l'instant éparses, insuffisantes voire inexistantes. Ce travail vise à combler ce gap à travers une analyse de la diversité, des paramètres de croissance et d'exploitabilité de la faune piscicole de ce réservoir. Pour ce faire, les poissons ont été échantillonnés au filet maillant et épervier entre avril 2018 et janvier 2019. Les espèces ont été identifiées et chaque poisson a été pesé puis mesuré. Au total 39 espèces ont été recensées et regroupées en 29 genres et 14 familles. Les familles les plus représentées sont Cichlidae, Mormyridae et Mochokidae. Les espèces dominantes sont Sarotherodon galilaeus Siluranodon auratus, Schilbe intermedius, Coptodon zillii et Oreochromis niloticus. L'étude suggère que les poissons et cet écosystème aquatique sont dans de bonnes conditions. Les résultats de cette étude contribueront à mieux planifier la gestion de cette nouvelle pêcherie et constitueront une base pour comprendre les changements futurs.

Mots-clés : Poissons, diversité, croissance, réservoir de Samandeni, Burkina Faso

\section{Abstract :}

Fish diversity and demographic parameters of the main fish species in the Samandeni reservoir, Burkina Faso.

The management of the Samandeni fishery, Burkina Faso, built in 2017 requires, reliable information but as for now, they are missing, insufficient and fragmentary. This piece of work intends to analyse the fish community in terms of diversity, growth, and exploitability. To do so, fish were sampled between April 2018 and January 2019 with cast net and gillnets. The species were identified, and each fish weighed, and its total length measured. We recorded 40 species, 
Diversité piscicole et paramètres démographiques des principales espèces de p...

regrouped in 29 genera and 14 families. The most represented families are the Cichlidae, Mormyridae and Mochokidae. Dominant species are Sarotherodon galilaeus Siluranodon auratus, Schilbe intermedius, Coptodon zillii et Oreochromis niloticus. The study suggests that fish and the aquatic ecosystem are in conditions. The results of this study will contribute to plan the management of the new fishery and provides basis to understand future changes.

Keywords : Fish, diversity, growth, Samandeni reservoir, Burkina Faso

\section{Introduction}

La faune piscicole a une importance pour l'homme à plusieurs titres. Premièrement, elle constitue une source nutritionnelle en protéines de qualité en plus d'être pourvoyeuse de nombreux micronutriments essentiels, y compris plusieurs vitamines (D, A et B), des minéraux (calcium, iode, zinc, fer et sélénium) et des acides gras polyinsaturés oméga-3 (Dibala et al., 2018). Deuxièmement, elle a une importance écologique car sa position dans la chaîne trophique est révélatrice de la qualité des rares eaux de surface des pays sahéliens de l'hinterland comme le Burkina Faso (Poda et al., 2004).

L'irrégularité des pluies, l'évaporation considérable des eaux de surface et l'importante croissance démographique du Burkina Faso qui est de l'ordre de 3\% par an, entrainent une insuffisance de l'eau au point d'hypothéquer ses possibilités de développement (Belem et al., 2018). L'analyse des indicateurs de pauvreté monétaire de 2004 indique que 40,1\% de la population du Burkina Faso vit en-dessous du seuil absolu de pauvreté avec un revenu annuel estimé à 153530 francs CFA (Burkina Faso, 2016). Cette situation est essentiellement due à l'avancée du désert et aux récurrentes difficultés d'accès à l'eau (Traoré, 2012).

Comme solution à la problématique de l'eau en tant que source et ressource, le Burkina Faso a opté depuis les années 1950 pour l'édification de réservoirs (Baijot et al., 1994). Actuellement, son potentiel halieutique, qualifié d'inattendu par Cecchi (2009) est essentiellement dû aux plans d'eau artificiels dont le nombre est estimé à plus de 1500 (Ouédraogo, 2010) avec une production halieutique qui augmenterait en moyenne de 200 à 250 tonnes par an (Cecchi, 2009) et de façon proportionnelle à la création de nouveaux réservoirs. C'est le récent cas du réservoir de Samandeni qui a augmenté la production nationale en poisson de 500 à 1000 tonnes (PHIE, 2019).

Plusieurs auteurs ont apporté leurs contributions à la connaissance de la faune piscicole du Burkina Faso vu son importance. Il s'agit entre autres des travaux d'inventaire qui ont permis de connaître davantage cette faune (Roman, 1966 ; Baijot et al., 1994 ; Sirima et al., 2009 ; Mano et al., 2019). D’autres études sur les poissons notamment sur les parasites (Kabré et al., 2002) sur le régime alimentaire (Toguyeni et al., 1996 ; Ouéda et al., 2008), et quelques paramètres biologiques (Melcher et al., 2012 ; Da et al., 2018 ; Minoungou et al., 2020) ont également fourni des données utiles aux aménagistes de la pêche.

Malheureusement, la diversité, les paramètres de croissance et d'exploitabilité des poissons du Mouhoun supérieur, lieu d'implantation du réservoir de Samandeni, n'ont jusque-là pas fait l'objet d'étude. De plus, ce réservoir qui vient d'être mis en eau, offre l'opportunité de faire l'état des lieux de la faune piscicole d'un réservoir sahélo-soudanien juste après sa mise en eau. Le but de cette étude est donc de générer des informations nécessaires à la modélisation des données piscicoles du 
réservoir. Ce qui permettra ultérieurement d'apprécier l'évolution de la biologie et l'écologie des poissons dans le temps. Ainsi, deux objectifs spécifiques sont visés à travers cette étude à savoir (i) faire l'inventaire de la faune ichtyologique du réservoir de Samandeni juste après sa mise en eau et (ii) déterminer les paramètres démographiques des espèces dominantes de ce réservoir.

\section{Méthodologie}

\section{Site d'étude}

La zone d'étude est le réservoir de Samandeni (figure 1), situé entre les longitudes $11^{\circ} 23^{\prime} 55,90^{\prime \prime}$ $\mathrm{N}$ et $11^{\circ} 19^{\prime} 59,83^{\prime \prime} \mathrm{N}$ et les latitudes $004^{\circ} 34^{\prime} 41,40^{\prime \prime} \mathrm{W}$ et $004^{\circ} 46^{\prime} 00,14^{\prime \prime} \mathrm{W}$. D'une capacité de 15000 ha de superficie inondée, le réservoir de Samandeni est le troisième plus grand réservoir du Burkina Faso après ceux de la Kompienga et de Bagré (Yonkeu et al., 2009). Sa construction s'est achevée par sa mise en eau le 11 mai 2017. Cet important périmètre halieutique d'intérêt économique de la région de l'Ouest du Burkina Faso, défini comme tel par le Décret n॰2012-1065/ PRES/PM/MEDD/MATDS du 31 décembre 2012 (Présidence du Faso, 2014), permettra à terme, la création d'une zone agro-industrielle ainsi que l'aménagement de 21000 hectares de périmètre irrigué en maitrise totale d'eau (Attakin, 2006).

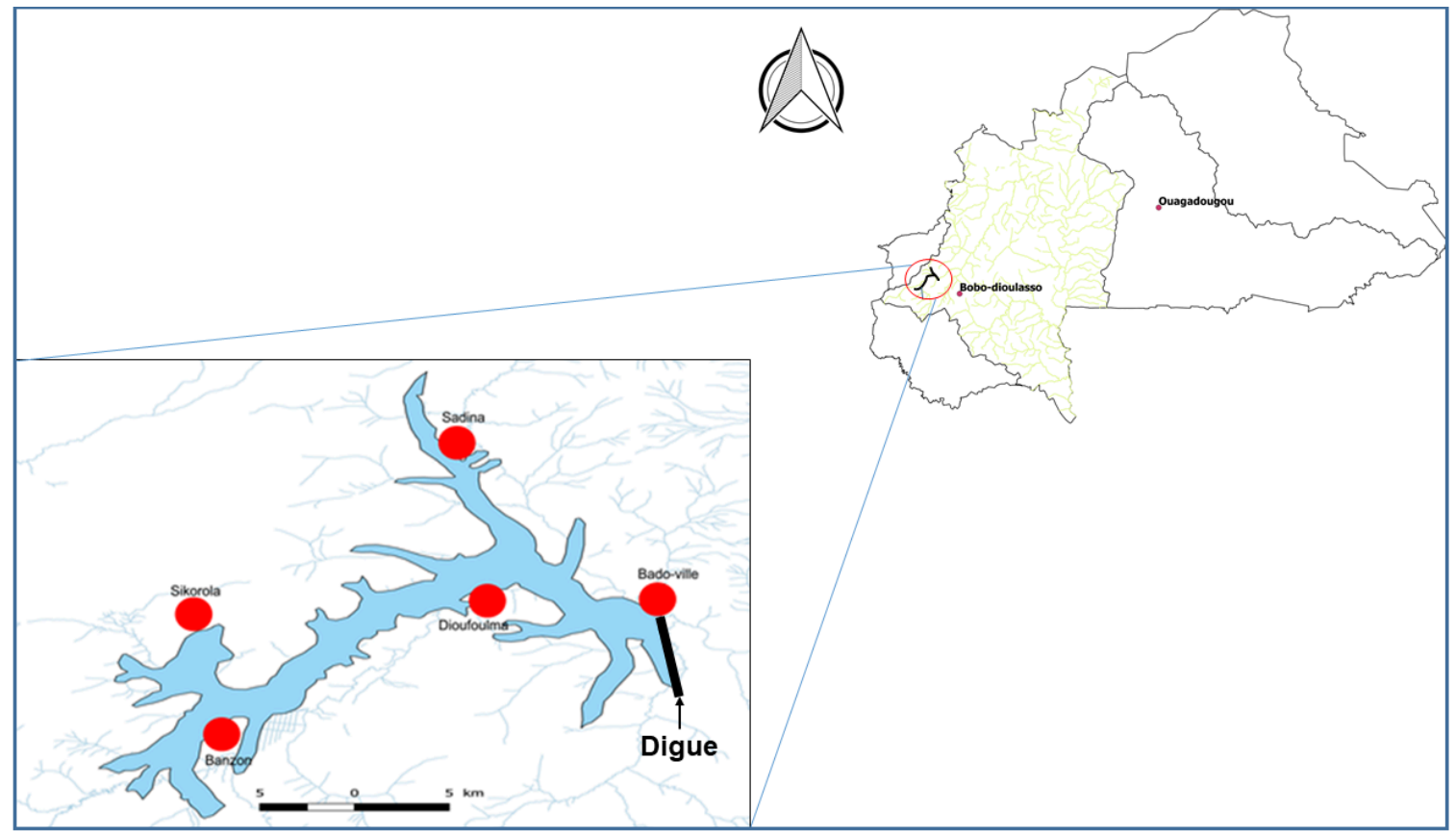

Figure 1 : Site d'étude, le résevoir de Samandeni au Burkina Faso, avec les stations d'échantillonnage en rouge

Cinq stations d'échantillonnage ont été choisies suivant le gradient longitudinal du réservoir afin d'avoir sa meilleure couverture. Il s'agit des stations de Bado-ville (11 ${ }^{\circ} 23^{\prime} 55,90^{\prime \prime} \mathrm{N}$; 004 ${ }^{\circ} 34^{\prime} 41,40^{\prime \prime}$

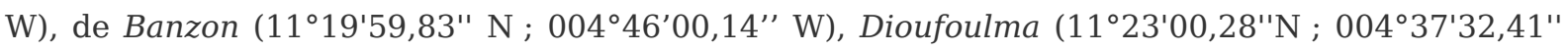

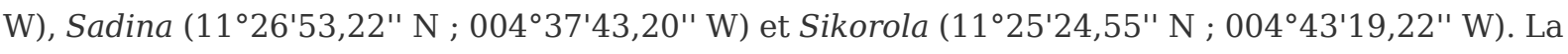
station de Bado-ville est située au niveau de la digue du réservoir. Elle est à $24 \mathrm{~km}$ à vol d'oiseau des stations les plus en amont (Sikorola et Bazon). 
Diversité piscicole et paramètres démographiques des principales espèces de $\mathrm{p} .$.

\section{Collecte des données}

Les poissons ont été échantillonnés lors de pêches expérimentales au moyen de filet épervier, maille de $15 \mathrm{~mm}$ de côté et de filets maillants, mailles de 20, 30, 45, 60 et $65 \mathrm{~mm}$ de côté. Les filets maillants utilisés dans cette étude sont des filets multi-filaments dont la ralingue supérieure est munie de flotteurs et de plombs au niveau de la ralingue inférieure. Deux pêcheurs professionnels ont été recrutés pour ces pêches expérimentales qui ont été réalisées à pied et accessoirement en pirogue en fonction de la profondeur de la zone prospectée. L'échantillonnage a eu lieu entre avril 2018 et janvier 2019 avec quatre campagnes d'échantillonnage, respectivement en avril, juillet, novembre 2018 et janvier 2019. Pendant cette période, le plan d'eau n'était pas encore ouvert à la pêche. Les filets maillants ont été posés entre $16 \mathrm{~h}$ et $17 \mathrm{~h}$ et relevés le lendemain entre $08 \mathrm{~h}$ et 09h. Plus d'une centaine de pose des filets ont été effectués par station d'échantillonnage et par campagne.

L'identification des espèces a été faite à l'aide de la clé d'identification de Paugy et al. (2003) d'abord sur le terrain quand c'est possible. Sinon, les spécimens sont conservés sous alcool à $70 \%$ et transportés au Laboratoire de Biologie et Écologie Animales (LBEA) de l'Université Joseph KIZERBO ou au Laboratoire d'Histoire Naturelle du CNRST pour détermination.

Les longueurs totales (LT) en cm et le poids (PT) en g ont été respectivement mesurées grâce à un ichtyomètre gradué à $1 \mathrm{~mm}$ près et une balance électronique d'une précision de 0,1 g.

\section{Analyse de la diversité ichtyologique}

L'indice de diversité de Shannon-Weaver ( $\left.\mathrm{H}^{\prime}\right)$ et l'indice d'équitabilité de Piélou ( $\left.E^{\prime}\right)$ ont été utilisés pour analyser la diversité du peuplement ichtyologique. Le diagramme de Venn a été utilisé pour mettre en évidence les espèces spécifiques et celles communes aux deux saisons de la zone d'étude.

\section{Croissance, mortalité et exploitabilité des principales espèces}

FiSAT II a été utilisé pour estimer les paramètres de croissance, la mortalité et l'exploitabilité de sept espèces dominantes d'intérêt économique du réservoir. Ainsi, le choix de ces espèces a été motivé par leur abondance relative. A cet effet, les paramètres suivants ont été estimés : le coefficient de croissance de Von Bertalanffy $(\mathrm{K})$, la longueur asymptotique $\left(L_{\infty}\right)$, la mortalité totale $(Z)$, la mortalité naturelle $(M)$, la mortalité par pêche $(F)$ et l'exploitabilité $(E)$.

Les données de distributions des fréquences de taille ont été utilisées pour estimer, de deux manières différentes, les paramètres de croissance $K$ et $L_{\infty}$ suivant l'équation de von Bertalanffy : (1) par la méthode d'ELEFAN I (Electronic LEngth Frequency ANalysis) décrite dans Pauly (1997) et (2) par la méthode de la courbe de croissance linéaire. En définitive, les moyennes des $\mathrm{K}$ et $L_{\infty}$ issues des deux méthodes ont été utilisées pour apprécier la croissance linéaire de chacune des espèces étudiées.

$\mathrm{Z}$ a été calculé sur la base du nombre de survivants de chaque espèce en fonction du temps selon la formule de Gulland (1969) ci-dessous dans laquelle $\Delta N_{t}$ représente la variation de l'effectif des poissons, $\Delta t$ la variation de temps et $N_{t}$ est le nombre d'espèces au temps « $\mathrm{t} »$ : 


$$
\frac{\Delta N_{t}}{\Delta t}=-Z * N_{t}
$$

Si l'on considère que $Z$ est constante toute une vie durant pour chacune des espèces étudiées, l'équation de la mortalité $\mathrm{Z}$ devient :

$$
N_{t}=N_{0} * e^{Z_{t}\left(t-t_{0}\right)}
$$

La mortalité naturelle (M) a été calculée par la méthode de Pauly (1980) qui repose sur la relation entre $\mathrm{M}, \mathrm{K}, \mathrm{L}_{\infty}$ et la température du milieu selon la formule ci-dessous. La température annuelle moyenne utilisée ici est de $30^{\circ} \mathrm{C}$. Elle correspond aux mesures réalisées sur le terrain pendant la période de l'étude.

$$
\log (M)=a+\operatorname{blog}\left(L_{\infty}\right)+\operatorname{clog}(K)+\operatorname{dlog}(T)
$$

avec :

$$
a=-0,21 ; b=-0,0824 ; c=0,6757 ; d=0,4627
$$

Quant à la mortalité par pêche (F) qui est le ratio des captures sur la biomasse, elle a été estimée en faisant, car la mortalité totale est la somme des mortalités naturelle (M) et par pêche (F).

Enfin, l'exploitabilité (E) a été estimée par la relation ci-dessous d'après Pauly (1985) :

$$
E=F /(F+M)=F / Z
$$

\section{Résultats}

\section{Diversité piscicole}

Au total 39 espèces de poisson ont été identifiées au cours de cette étude (tableau 1). Ces espèces se regroupent en 29 genres et 14 familles. Les familles des Mormyridae (7 espèces), des Cichlidae (6 espèces) et des Mochokidae (5 espèces) sont les plus représentées. Le nombre d'espèces 
Diversité piscicole et paramètres démographiques des principales espèces de p...

rencontrées décroît de la digue vers la partie haute du réservoir : 36 espèces à Bado-ville (digue) ; 19 espèces à Dioufoulma ; 14 espèces à Sadina ; 16 espèces à Sikorola et 10 espèces à Banzon.

Tableau 1 : Abondance relative par ordre décroissant, poids et taille moyenne et occurrence des espèces capturées dans le réservoir de Samandeni au Burkina Faso

\begin{tabular}{|c|c|c|c|c|c|c|c|c|c|c|c|c|c|}
\hline & & & & & & & & & tation & & & & ons \\
\hline Cichlidae & Sarotherodon & Sarotherodon galilaeus (Linnaeus, 1758) & 590 & 16.93 & 17,53 & 88,94 & $*$ & * & $*$ & * & $*$ & $*$ & * \\
\hline Mochokidae & Synodontis & Synodontis sp. & 468 & 13.43 & 14,98 & 62,39 & * & & & & & * & \\
\hline Schilbeidae & Siluranodon & Siluranodon auritus (Geoffroy Saint-Hilaire, 1809) & 454 & 13.03 & 53,66 & 4,08 & $*$ & & & & & * & \\
\hline Schilbeidae & Schilbe & Schilbe intermedius, Rüppell 1832 & 331 & 9.50 & 19,66 & 57,76 & * & & $*$ & * & * & * & * \\
\hline Cichlidae & Coptodon & Coptodon zillii (Gervais, 1848) & 229 & 6.57 & 14,63 & 77,18 & * & * & * & $*$ & $*$ & * & $*$ \\
\hline Cichlidae & Oreochromis & Oreochromis niloticus (Linnaeus, 1758) & 195 & 5.60 & 14,47 & 73,02 & * & $*$ & * & * & * & * & * \\
\hline Mochokidae & Synodontis & Synodontis schall (Bloch \& Schneider, 1801) & 171 & 4.91 & 19,91 & 83,82 & * & & * & * & * & ${ }^{*}$ & * \\
\hline Alestidae & Brycinus & Brycinus nurse ((Rüppell, 1832)) & 140 & 4.02 & 15,74 & 41,27 & $*$ & $*$ & * & & & * & * \\
\hline Claroteidae & Chrysichthys & Chrysichthys auratus (Geoffroy Saint-Hilaire, 1809) & 106 & 3.04 & 18,73 & 91,03 & * & & * & * & & * & * \\
\hline Cichlidae & Hemichromis & Hemichromis fasciatus, Peters 1857 & 99 & 2.84 & 15,04 & 69,05 & * & * & * & * & * & * & * \\
\hline Mormyridae & Petrocephalus & Petrocephalus bovei (Valenciennes, 1847) & 62 & 1.78 & 8,50 & 6,22 & * & & & & & * & \\
\hline Cyprinidae & Labeo & Labeo coubie, Rüppell, 1832 & 43 & 1.23 & 38,16 & 663,47 & * & & * & * & $*$ & * & * \\
\hline Cyprinidae & Enteromius & Enteromius macrops (Boulenger, 1911) & 36 & 1.03 & 7,75 & 5,52 & $*$ & & & & $*$ & $*$ & $*$ \\
\hline Schilbeidae & Parailia & Parailia pellucida (Boulenger, 1901) & 33 & 0.95 & 6,27 & 0,98 & * & & & & & $*$ & \\
\hline Mochokidae & Synodontis & Synodontis punctifer, Daget, 1965 & 31 & 0.89 & 18,30 & 65,52 & $*$ & & $*$ & & $*$ & & * \\
\hline Mormyridae & Mormyrus & Mormyrus rume, Valenciennes, 1847 & 19 & 0.55 & 39,39 & 664,14 & * & & * & & * & * & * \\
\hline Mochokidae & Synodontis & Synodontis filamentosus, Boulenger, 1901 & 10 & 0.29 & 19,77 & 69,50 & $*$ & & & & & * & \\
\hline Mormyridae & Hippopotamyrus & Hippopotamyrus pictus (Marcusen, 1864) & 10 & 0.29 & 26,73 & 168,14 & * & & * & * & & & * \\
\hline Mormyridae & Hyperopisus & Hyperopisus bebe (Lacépède, 1803) & 10 & 0.29 & 28,35 & 198,16 & * & & & * & & * & * \\
\hline Cichlidae & Chromiditilapia & Chromidotilapia güntheri (Sauvage, 1882) & 8 & 0.23 & 11,35 & 25,41 & * & & & & & & * \\
\hline Cichlidae & Hemichromis & Hemichromis bimaculatus, Gill, 1862 & 8 & 0.23 & 7,24 & 5,86 & & * & & & * & & * \\
\hline Cyprinidae & Leptocypris & Leptocypris niloticus (Joannis, 1835) & 8 & 0.23 & 6,26 & 1,94 & * & & & & & * & \\
\hline Alestidae & Brycinus & Brycimus macrolepidotus, Valenciennes, 1850 & 5 & 0.14 & 26,71 & 118,29 & * & ${ }^{*}$ & & & & * & * \\
\hline Arapaimidae & Heterotis & Heterotis niloticus (Cuvier, 1829) & 3 & 0.09 & 37,63 & 572,67 & & & * & & $*$ & ${ }^{*}$ & $*$ \\
\hline agridae & Bagrus & Bagrus docmak (Forsskål, 1775) & 3 & 0.09 & 40,7 & 140,34 & & & & * & & * & \\
\hline Gymnarchidae & Gymnarchus & Gymnarchus niloticus, Cuvier, 1829 & 3 & 0.09 & 61,43 & 116,62 & & * & * & & & & * \\
\hline Mochokidae & Synodontis & Synodontis clarias (Linnaeus, 1758) & 3 & 0.09 & 23,6 & 171,2 & $*$ & & & & & & * \\
\hline Mormyridae & Mormyrops & Mormyrops anguilloides (Linnaeus, 1758) & 3 & 0.09 & 39,07 & 384,7 & * & & & & & & * \\
\hline Malapteruridae & Malapterurus & Malapterurus electricus (Gmelin, 1789) & 2 & 0.06 & 24,25 & 204,52 & $*$ & & & & & * & * \\
\hline Cyprinidae & Chelaethiops & Chelaethiops bibie (Joannis, 1835) & 1 & 0.03 & 8,2 & 2,33 & $*$ & & & & & & $*$ \\
\hline Anabantidae & Ctenopoma & Ctenopoma kingsleyae, Günther, 1896 & 1 & 0.03 & 14,7 & 48,0 & * & & & & & * & \\
\hline Mochokidae & Synodontis & Synodontis membranaceus (Geoffroy Saint-Hilaire, 1809) & 1 & 0.03 & 38.00 & 604.00 & * & & & & & * & \\
\hline Mormyridae & Brienomyrus & Brienomyrus niger (Günther, 1866) & 1 & 0.03 & 12.00 & 16.00 & * & & & & & * & \\
\hline
\end{tabular}

Nbre sp. : Nombre de spécimens ; L.m. : longueur moyenne ; P.m. : poids moyen ; B-v. : Station de Bado-ville ; Ban. : Station de Banzon; Dio. : Station de Dioufoulma ; Sad : Satation de Sadina ; Sik. : Station de Sikorola ; S.P. : Saison Pluvieuse ; S.S : Saison Sèche. *: Présence

$\mathrm{Au}$ total, 30 espèces ont été rencontrées en saison sèche (janvier et avril) et 31 en saison pluvieuse (juillet et novembre). Vingt deux (22) espèces étaient communes aux deux saisons, 9 espèces exclusivement rencontrées en saison pluvieuse et 8 espèces en saison sèche (figure 2). 

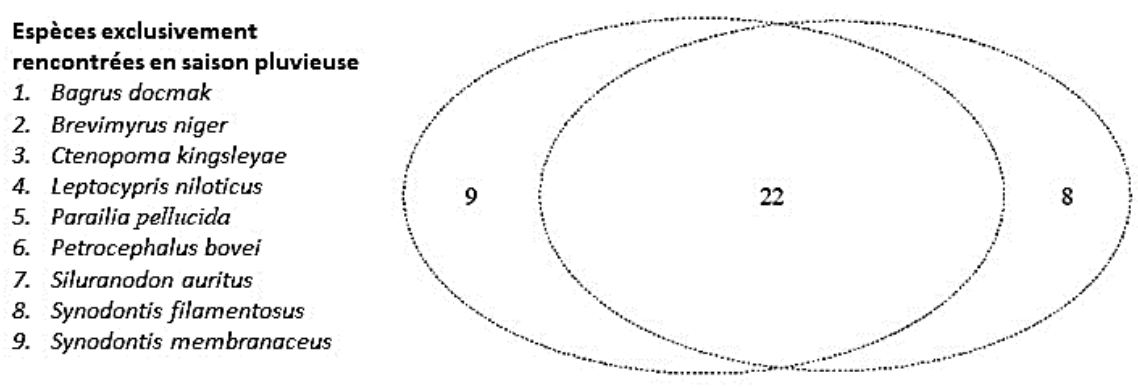

Espèces exclusivement

rencontrées en saison sèche

1. Chelaethiops bibie

2. Chromidotilapia giuntheri

3. Gymnarchts niloticus

4. Hemichromis bimaculatus

5. Hippopotamyrus pictus

6. Mormyrops anguilloides

7. Synodontis clarias

8. Synodontis punctifer

Figure 2 : Distribution des espèces de poissons capturées par saison le résevoir de Samandeni au Burkina Fasod’avril 2018 à janvier 2019

Sur les 3484 spécimens de poissons pêchés, Sarotherodon galilaeus est l'espèce la plus abondante (tableau 2), suivie de Siluranodon auratus, Schilbe intermedius, Coptodon zillii, Oreochromis niloticus, Marcusenius senegalensis, Synodontis schall, Brycinus nurse et les autres.L'indice de Shannon pour les poissons capturés dans le réservoir de Samandeni pris dans sa globalité, indique une diversité supérieure à la moyenne classique $\left(3<\mathrm{H}^{\prime}<4\right)$ aussi bien en saison pluvieuse qu'en saison sèche. Les indices d'équitabilité de Pielou ( $\left.E^{\prime}\right)$ révèlent une régularité du peuplement piscicole se traduisant ainsi par des valeurs de E qui tendent vers 1 (Tableau 2).

Tableau 2 : Richesse spécifique, indice de Shannon et d'équitabilité de Pielou des poisons du réservoir de Samandeni par saison

\begin{tabular}{lll}
\hline Indices & Saison Pluvieuse & Saison sèche \\
\hline Richesse spécifique (S) & 31 & 30 \\
Indice de Shannon-Weaver (H') & 3,78 & 3,46 \\
Equitabilité de Pielou (E') & 0,76 & 0,7 \\
Hmax & 4,95 & 4,91 \\
\hline
\end{tabular}

\section{Croissance, mortalité et exploitabilité des principales espèces}

Le tableau 3 récapitule les valeurs des paramètres de croissance $\mathrm{K}$ et de Von Bertalanffy obtenues à partir de la méthode K-scan de ELEFAN 1 et celles obtenues par les courbes de croissance linéaire. Les valeurs moyennes des $\mathrm{K}$ indiquent que Marcusenius senegalensis est l'espèce qui croît le plus vite vers sa longueur asymptotique qui est de 31,4 cm avec une croissance annuelle $\mathrm{K}$ de 1,17 cm. Elle est suivie par Coptodon zillii $(0,95 \mathrm{~cm} / \mathrm{an})$, Brycinus nurse $(0,61 \mathrm{~cm} / \mathrm{an})$, Schilbe intermedius $(0,58 \mathrm{~cm} / \mathrm{an})$, Synodontis schall $(0,56 \mathrm{~cm} / \mathrm{an})$, Sarotherodon galilaeus $(0,49 \mathrm{~cm} / \mathrm{an})$ et Oreochromis niloticus $(0,49 \mathrm{~cm} / \mathrm{an})$. 
Diversité piscicole et paramètres démographiques des principales espèces de p...

Tableau 3 : Paramètres de croissance $K$ et $L_{\infty}(\mathrm{cm})$

\begin{tabular}{|c|c|c|c|c|c|c|c|c|c|c|c|c|c|}
\hline \multirow[t]{2}{*}{ Espèces } & \multirow[t]{2}{*}{$\mathrm{N}$} & \multicolumn{3}{|c|}{ Longueur (cm) } & \multicolumn{3}{|c|}{ Poids (g) } & \multicolumn{2}{|c|}{ K-scan $(\mathrm{cm})$} & \multicolumn{2}{|c|}{ CCL $(\mathrm{cm})$} & \multicolumn{2}{|c|}{$\begin{array}{l}\text { Moyenne } \\
(\mathrm{cm})\end{array}$} \\
\hline & & Min & Moy & Max & Min & Moy & Max & $\begin{array}{l}\mathrm{K} \\
(\mathrm{an}-1)\end{array}$ & & $\begin{array}{l}\mathrm{K} \\
(\mathrm{an}-1)\end{array}$ & & $\begin{array}{l}\mathrm{K} \\
(\mathrm{an}-1)\end{array}$ & \\
\hline $\begin{array}{l}\text { Sarotherodon } \\
\text { galilaeus }\end{array}$ & 590 & 4 & 17,71 & 28,7 & 0,5 & 119,88 & 421,6 & 0,72 & 30,45 & 0,27 & 30 & 0,5 & 30,23 \\
\hline Schilbe intermedius & 331 & 6,1 & 17,92 & 31,2 & 1 & 44,5 & 264,2 & 0,8 & 32,66 & 0,36 & 34,76 & 0,58 & 33,71 \\
\hline Coptodon zillii & 229 & 1,5 & 15,25 & 23,6 & 0.076 & 82,62 & 256,8 & 1,3 & 25,73 & 0,59 & 20 & 0,95 & 22,87 \\
\hline $\begin{array}{l}\text { Marcusenius } \\
\text { senegalensis }\end{array}$ & 196 & 11,2 & 15,61 & 29,6 & 13,7 & 85,5 & 547 & 1,8 & 31,71 & 0,55 & 31,14 & 1,18 & 31,43 \\
\hline $\begin{array}{l}\text { Oreochromis } \\
\text { niloticus }\end{array}$ & 195 & 1,8 & 15,61 & 26,7 & 6,9 & 85,5 & 240,19 & 0,53 & 28,14 & 0,46 & 22 & 0,5 & 25,07 \\
\hline Synodontis schall & 171 & 13,4 & 22,92 & 35,5 & 30,9 & 129,49 & 591 & 0,61 & 38,33 & 0,52 & 32,53 & 0,57 & 35,43 \\
\hline Brycinus nurse & 140 & 6,5 & 15,74 & 26,7 & 1 & 41,27 & 160,5 & 0,74 & 28,88 & 0.48 & 25 & 0,61 & 26,94 \\
\hline
\end{tabular}

K-scan : Scanning of K-values ; CCL : Courbe de croissance linéaire

La figure 4 montre les graphiques correspondant aux estimations des coefficients de la mortalité totale ( $\mathrm{Z})$, naturelle $(\mathrm{M})$ et par pêche $(\mathrm{F})$ fondées sur la composition moyenne en âge de chacune des 7 espèces étudiées. Selon ces graphiques, Oreochromis niloticus a la plus faible mortalité totale $\left(2,57 \mathrm{an}^{-1}\right)$. La mortalité totale la plus élevée est observée chez Schilbe intermedius $\left(7,86 \mathrm{an}^{-1}\right)$. Les résultats révèlent des taux d'exploitation maximale $(E>0,5)$ pour toutes les espèces étudiées à l'exception de Marcusenius senegalensis $(\mathrm{E}=0,26)$ se traduisant par des coefficients de mortalité par pêche (F), supérieurs aux coefficients de mortalité naturelle (M) (Tableau 4). 
Tropicultura Tropicultura 2295-8010 Volume 39 (2021) Numéro 4, 1948

Tableau 4 : Mortalité naturelle (M), par pêche (F) et totale (Z) et niveau d'exploitation (E) des espèces étudiées

\begin{tabular}{lccccc}
\hline Espèces & M & F & Z & & E \\
\hline Sarotherodon galilaeus & 1,48 & 4,2 & 5,68 & 30,23 & 0,74 \\
Schilbe intermedius & 1,56 & 6,3 & 7,86 & 33,71 & 0,8 \\
Coptodon zillii & 2,29 & 2,36 & 4,64 & 22,87 & 0,51 \\
Marcusenius senegalensis & 2,67 & 0,91 & 3,58 & 31,43 & 0,26 \\
Oreochromis niloticus & 1,24 & 1,33 & 2,57 & 25,07 & 0,52 \\
Synodontis schall & 1,25 & 2,16 & 3,41 & 35,43 & 0,63 \\
Brycinus nurse & 1,53 & 2,89 & 4,42 & 26,07 & 0,65 \\
\end{tabular}

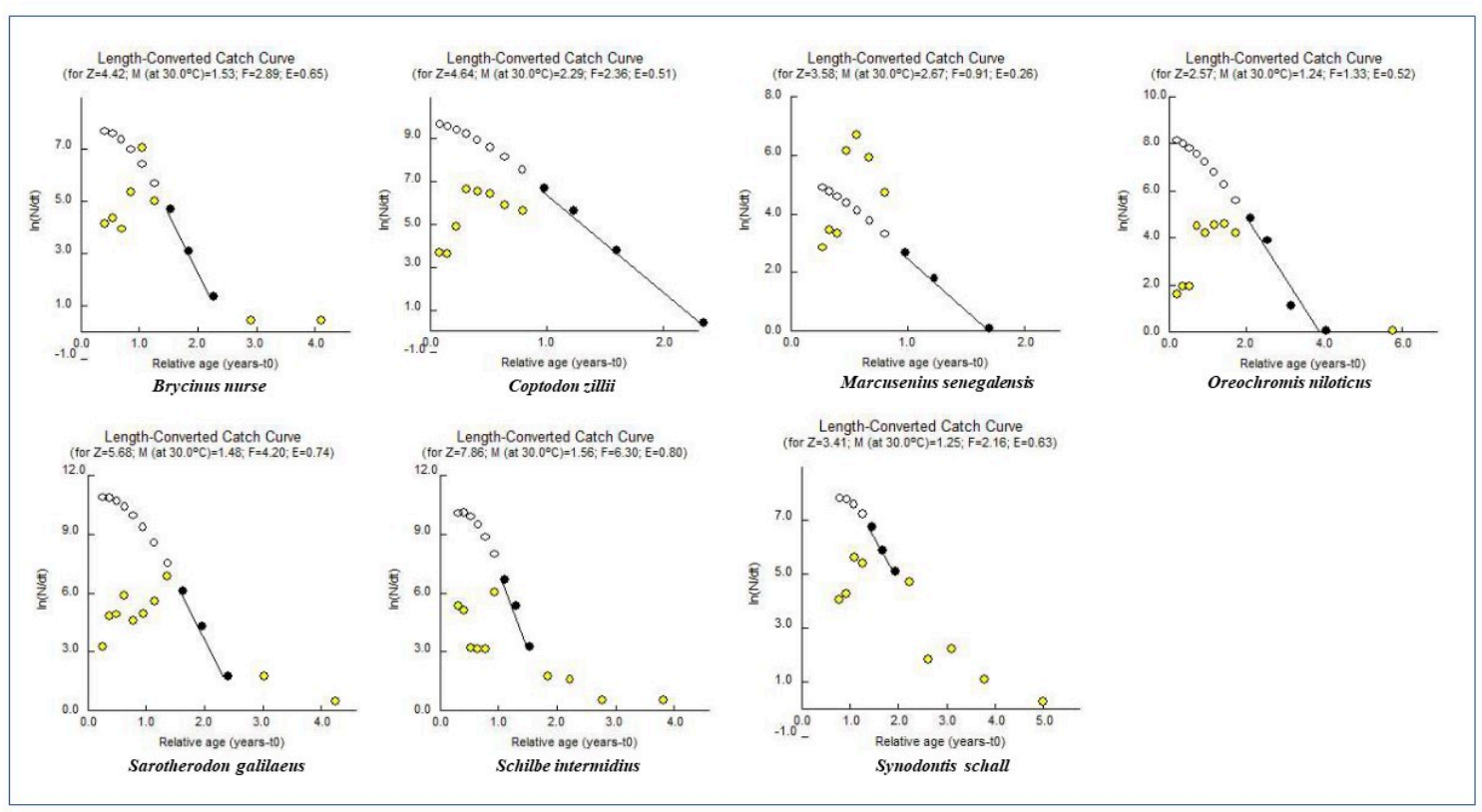

Figure 3 : Mortalités ( $Z=$ mortalité, $M=$ mortalité naturelle, $F=$ mortalité par pêche) et niveau d'exploitation (E) des espèces étudiées pour une température de $30{ }^{\circ} \mathrm{C}$

\section{Discussion}

\section{Diversité piscicole}

Le réservoir de Samandeni compte pour le moment et selon les résultats de nos travaux d’inventaire, 
Diversité piscicole et paramètres démographiques des principales espèces de $\mathrm{p} .$.

39 espèces réparties dans 29 genres et 14 familles. Cela représente plus de la moitié des espèces de poisson récemment répertoriées par Mano et al. (2019) à l'issue des travaux d'assemblage des poissons du Nakanbé et du Mouhoun, deux affluents de la Volta. Cette zone qui couvre 64\% du territoire Burkinabè compterait d'après cette étude, 79 espèces de poisson regroupées en 41 genres et 19 familles. Le réservoir de Bagré, l'une des 9 stations d'échantillonnage concernées par les travaux de Mano et al. (2019) et dont les paramètres associés à la taille (débit, matières transportées, etc.) sont proches de ceux du réservoir de Samandeni, compterait 42 espèces. Selon Zampaligre (2004) cité par Ouédraogo (2010), le réservoir de Kompienga, semblable aux précédents, compterait 34 espèces de poisson.

Les travaux de Ouédraogo (2010) ont également concerné le lac Bam (1400 ha), certes relativement petit par sa taille, mais doté d'un barrage au niveau de son bassin versant comme les trois précédents réservoirs. Le lac Bam, ruiné par les effets anthropiques compterait 20 espèces (Ouédraogo, 2010). Par ailleurs, Baijot et al. (1994) ont trouvé que le lac de barrage de Loumbila, dont la superficie est de 1500 ha, comptait 26 espèces piscicoles. De cette étude, il ressort aussi que la réserve de biosphère de Bala (Mare aux hippopotames), compterait 28 espèces de poisson pour une superficie de 1200 ha (Baijot et al., 1994).

S'il est difficile de comparer la diversité piscicole de différentes eaux étant donné leurs caractéristiques souvent différentes, il est en revanche établi que le nombre d'espèces de poisson présentes dans une retenue d'eau est lié à sa taille et à l'ensemble des paramètres associés à sa taille (De Mérona et al., 2005). Ainsi, la relation calculée à partir des données transformées en logarithmes décimaux dans $\mathrm{R}$ de ces six plans d'eau du Burkina Faso est linéaire avec une corrélation assez significative de 76,55\% et p-value $=0,02248<0,05$ (Figure 5). Il apparaît que le réservoir de Samandeni, comme la Mare aux Hippopotames, se situe au-dessus de la relation moyenne et donc, relativement à sa taille, serait plus riche en espèces que la moyenne des autres réservoirs. Cela suggère que, le réservoir de Samandeni, comme la Mare aux Hippopotames, est moins impacté que les plans d'eau de Kompienga, Bam et Loumbila.

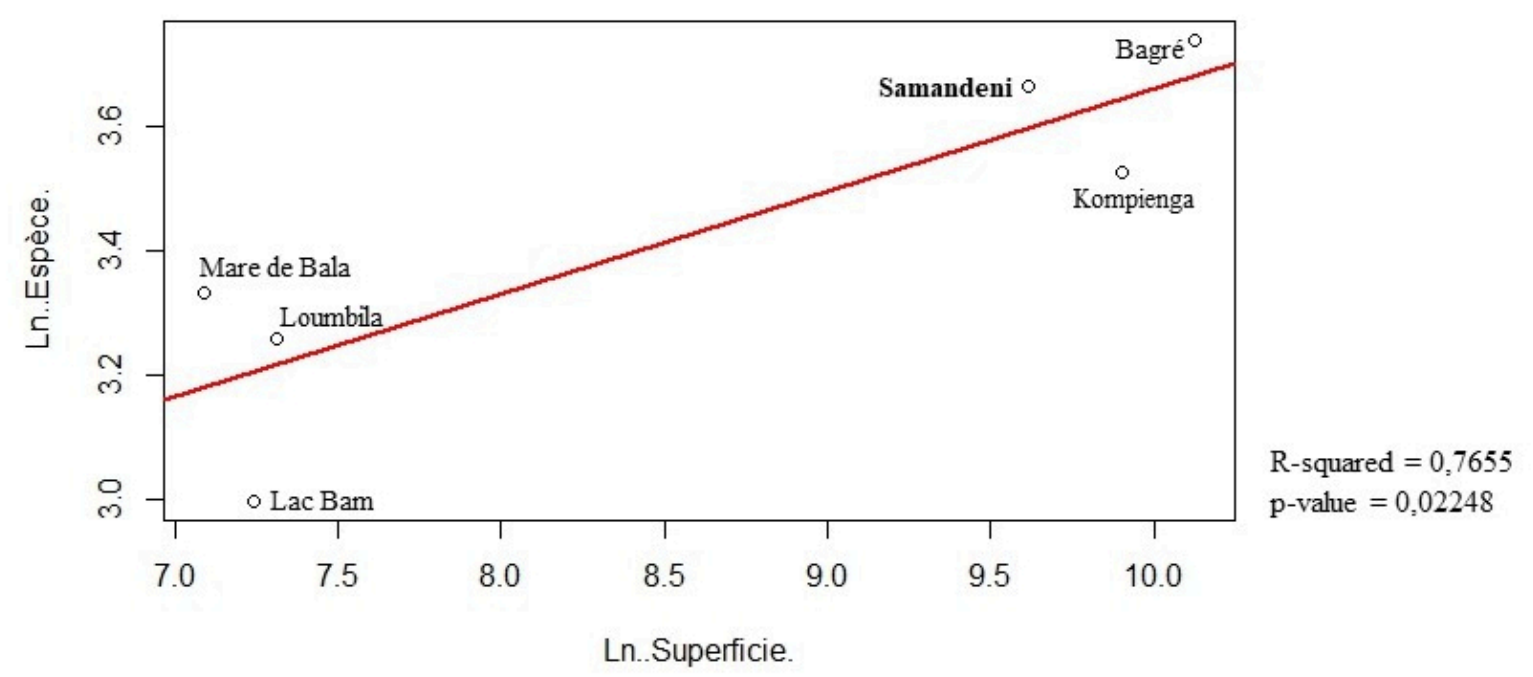

Figure 4 : Relation entre superficies et nombre d'espèces de poisson de quelques plans d'eau du Burkina Faso (Données en logarithmes décimaux). 
En se fondant également sur la relation établie par Hugueny (1989) entre richesse spécifique et surface des bassins versants d'Afrique de l'Ouest, théorie utilisée par Lalèyè et al. (2004) pour apprécier la diversité ichtyologique du bassin du fleuve Ouémé au Bénin, on parvient à une prédiction de $\mathrm{N}=34$ espèces pour le réservoir de Samandeni étant attendu que la formule est la suivante : avec S la surface du bassin versant du Mouhoun supérieur (20978 ha) et c $(1,4163)$ la pente de l'équation linéaire mettant en relation les 6 plans d'eau.

D'après les dernières mises à jour de la base de données informatiques sur les poissons «FishBase », le Burkina Faso compterait 123 espèces de poissons (Froese R, 2019). Ces données sont à deux espèces près celles d'une ancienne étude réalisée par Roman (1966), répertoriant 121 espèces dans la partie supérieure de la Volta. Plusieurs raisons pourraient expliquer cet important gap entre le nombre d'espèces identifiées à Samandeni (39) et celui supposé être du Burkina Faso (123). Premièrement, les données de « FishBase » concernent le nombre d'espèces des trois bassins internationaux que compte le Burkina Faso à savoir le Mouhoun, le Niger et la Comoé. Alors que nos données ne concernent que celles du réservoir de Samandeni localisé dans la partie supérieure du Mouhoun. Deuxièmement, durant nos travaux, les pêches commerciales et sportives étaient interdites. Or, ces pêches fournissent d'importantes données complémentaires comme l'ont relevé certains auteurs tels que Sirima et al. (2009) et Ouédraogo (2010).

Cette différence pourrait également être due au déclin des espèces, causé par les obstructions des cours d'eau par les nombreux barrages que compte le Burkina Faso (plus de 1500 barrages) qui pour la plupart, y compris celui de Samandeni, ne sont pas munis de passes à poisson. A ces deux raisons, l'on pourrait ajouter la difficulté qu'il y a eu à couvrir la diversité des habitats sur l'étendue du réservoir de Samandeni comme le fait remarquer Bance et al. (2021). En effet, vu les prospections réalisées à diverses périodes de l'année et à différents endroits du réservoir, vraisemblablement, une bonne part des espèces a été identifiée. Toutefois, il serait prétentieux de penser que la méthodologie d'échantillonnage utilisée a permis d'identifier toutes les espèces d'un réservoir de l'envergure du lac de barrage de Samandeni car, les espèces ont des préférenda d'habitat, des aires de répartition différentes ainsi que des fluctuations saisonnières, du fait des phénomènes de recrutement et de mortalité (Lalèyè et al., 2004 ; De Mérona et al., 2005).

Des analyses quantitatives et qualitatives plus approfondies indiquent que les Mormyridae (7 espèces) ; les Cichlidae (6 espèces) et les Mochokidae (5 espèces) sont respectivement les familles les plus représentatives du réservoir. La prédominance des Mormyridae, peut être due à la position géographique du réservoir de Samandeni construit au confluent des eaux de la rivière Mouhoun, l'un des seuls cours d'eau permanent du Burkina Faso d'après Dakoure (2003) même si certains auteurs comme Ouédraogo (2010) remettent en cause la nature permanente de ce cours d'eau qui, par endroit et à certain moment de l'année, peut s'assécher totalement. Ce fut le cas en avril 2010 (Ouédraogo, 2010).

Les conditions presque naturelles $\mathrm{du}$ bassin du Mouhoun pourraient également expliquer l'abondance des espèces de cette famille comme le fait remarquer Sirima et al. (2009) pour justifier l'abondance des Mormyridae dans les eaux des aires protégées du bassin de la Comoé. En effet, le réservoir de Samandeni est situé au cœur du bassin du Mouhoun. Cette zone se caractérise par une végétation associée à de nombreuses forêts et zones humides d'intérêts écologiques d'environ 76000 hectares (Dibloni et al., 2009) au nombre desquels on peut citer la forêt galerie de la Guinguette (sous-Bassin du Kou), la Mare aux Hippopotames de Bala (Mouhoun supérieur), le cône d'épandage de Banh (Sourou) (Yonkeu et al., 2009). 
Diversité piscicole et paramètres démographiques des principales espèces de $\mathrm{p} .$.

Quant aux Cichlidae, de nombreuses études ont documenté la dominance des espèces de cette famille dans les rivières ouest-africaines (Lévêque, 1999; Lalèyè et al., 2004; Ouédraogo et al., 2015). Selon ces études, les espèces de cette famille constituent l'une des principales caractéristiques de la province ichtyologique nilo-soudanienne. Les trois Tilapia (S. galilaeus, C. zillii et O. niloticus) en particulier, possèderaient des aptitudes notamment une maturité précoce, une grande adaptabilité alimentaire, une tolérance environnementale élevées et, pour deux de ces espèces une particularité éthophysiologique d'incubateurs buccaux qui font d'elles des espèces piscicoles les mieux adaptées aux eaux tropicales ouest-africaines à faible niveau d'oxygène dissous.

La famille des Mochokidae, exclusivement représentée au sein du réservoir de Samandeni par des espèces du genre Synodontis, vient en troisième position après les Mormyridae et les Cichlidae. Des auteurs tel que Lauzanne (1988) soutiennent que dans les premiers moments de la création d'un lac de barrage comme celui de Samandeni, il se pose de grands changements dans l'éventail des ressources alimentaires en faveur des espèces ayant un large spectre de proies alimentaires telles que les espèces du genre Synodontis. Ces espèces sont connues pour leur régime alimentaire qui part du zooplancton aux détritus en passant par les insectes aussi bien aquatiques que terrestres (Lauzanne, 1988).

De plus, selon les travaux de Pinton (2008), Synodontis est, en termes d'abondance, le deuxième genre de poisson-chat des eaux douces d'Afrique. Les espèces du genre Synodontis sont également connues pour leur préférence des berges en herbes et en forêt de ces eaux (Danadu, 2012). Nos résultats se rallient à ces avis ainsi qu'à ceux de Gosse (1963) qui expliquent que les espèces de Synodontis font partie des poissons rhéophiles dont les habitats sont situés à proximité des rives. Ce qui pourrait expliquer sa grande abondance dans nos échantillons car la pêche se faisait principalement à pied, donc près des berges et accessoirement en pirogue.

\section{Paramètres démographiques}

Par comparaison, les tailles asymptotiques de Synodontis schall, Sarotherodon galilaeus, Coptodon zillii et Oreochromis niliticus obtenues à Samandeni sont supérieures à celles déjà rapportées (Baijot et al., 1994). Toutefois, ces tailles asymptotiques restent inférieures à celles rapportées ailleurs (Sirima et al., 2009), nonobstant des croissances annuelles parfois plus importantes à Samandeni (Tableau V). Ces résultats traduisent d'une part des conditions de vie plus ou moins favorables au sein du réservoir Samandeni pour ces espèces et d'autre part, une expérience de vie assez courte de celles-ci. Quant à Brycinus nurse, Schilbe intermedius et particulièrement Marcusenius senegalensis, leurs tailles asymptotiques sont supérieures à celles observées ailleurs.

En effet, plusieurs auteurs tel que Akombo et al. (2015), font remarquer que la croissance des poissons des eaux douces d'Afrique, en l'occurrence ces espèces pélagiques, est meilleure lorsque les conditions biotiques et abiotiques sont bonnes notamment lors de la montée des eaux. Tel est le cas à Samandeni avec la récente mise en eau du réservoir.

Malgré l'interdiction de la pêche durant la période de cette étude, à l'exception de $M$. senegalensis et dans une moindre mesure $C$. zillii, nos résultats indiquent des valeurs de E supérieures ou égales à 0,5 pour les six autres espèces étudiées, suggérant ainsi une exploitation maximale voire excédant le seuil critique d'exploitabilité pour $S$. intermedius $(0,8)$ en particulier (figure 4 ). Ces résultats suggèrent que le poisson était abondamment pêché avant la construction du barrage comme l'a déjà signalé Mano et al. (2019). 
Par ailleurs, lors des échantillonnages, des pêcheurs professionnels burkinabè et maliens y étaient clandestinement très actifs. Ils étaient très bien équipés par des mareyeurs venant de plusieurs horizons, y compris des grandes villes de Ouagadougou et Bobo-Dioulasso, avec beaucoup d'expérience sur les autres grandes pêcheries comme Bagré, Kompienga, Sourou et bien d’autres. Des pièges à poissons tels que des nasses, des filets maillants et des preuves de pêche clandestine des espèces facilement accessibles à partir des berges, ont été observés le long du réservoir (Minoungou et al., 2020). Tout cela pourrait expliquer le taux élevé de l'exploitation des espèces étudiées nonobstant le moratoire sur la pêche durant la période de l'étude.

Tableau 5 : Paramètres démographiques des 7 espèces dominantes de poisson du réservoir de Samandeni et d'autres eaux d'Afrique

\begin{tabular}{|c|c|c|c|c|c|}
\hline Espèce & Localité & Pays & $\mathbf{L}_{\infty}$ & $\mathbf{K}$ & Référence \\
\hline \multirow{5}{*}{ B. nurse } & Samandeni & Burkina Faso & 26,94 & 0,61 & Etude en cours \\
\hline & Moyen Niger & Niger & 19,06 & 0,80 & Daget, 1952 \\
\hline & Fleuve du Bandama & Côte d'Ivoire & 20,00 & 0,75 & Paugy 1980 \\
\hline & Bontanga reservoir & Ghana & 20,48 & 0,52 & Kwarfo-Apegyah et al., 2008 \\
\hline & Lac Tchad & Tchad & 14,70 & 0,65 & Benech et Quensière, 1987 \\
\hline \multirow{12}{*}{ O. niloticus } & Samandeni & Burkina Faso & 25,07 & 0,50 & Etude en cours \\
\hline & Okpara stream & Benin & 35,18 & 1,2 & Imorou et al., 2019 \\
\hline & Lac Ayamé I & Côte d'Ivoire & 35,50 & 0,48 & Tah et al., 2010 \\
\hline & Lac Victoria & Kenya & 61,30 & 0,35 & Getabu, 1992 \\
\hline & Tanguiga Reservoir & Burkina Faso & 17,60 & 0,46 & Baijot and Moreau 1997 \\
\hline & Dangouindougou & Burkina Faso & 22,00 & 0,50 & Sirima et al., 2009 \\
\hline & Bontanga reservoir & Ghana & 23,63 & 0,58 & Kwarfo-Apegyah et al., 2008 \\
\hline & Lac Tchad & Tchad & 45,17 & 0,32 & Blache 1964 \\
\hline & Lac Alaotra & Madagascar & 33,43 & 0,23 & Moreau 1979 \\
\hline & Lac Itasy & Madagascar & 57,21 & 0,14 & Moreau 1979 \\
\hline & Lac Mariut & Égypte & 42,59 & 0,46 & El Zarka et al. 1970 \\
\hline & Lac Albert & RD. Congo & 39,00 & 0,50 & Ssentongo 1971 \\
\hline \multirow[t]{3}{*}{ S. schall } & Samandeni & Burkina Faso & 35,43 & 0,57 & Etude en cours \\
\hline & Nil-Khartoum & Soudan & 86,02 & 0,11 & Bishai/Abu Gideiri, 1965 \\
\hline & Lower Benue river & Nigeria & 30,50 & 0,58 & Akombo et al., 2015 \\
\hline \multirow{7}{*}{ S. galilaeus } & Samandeni & Burkina Faso & 30,23 & $\mathbf{0 , 5 0}$ & Etude en cours \\
\hline & Bassin du Senegal & Senegal & 30,10 & 0,51 & Moreau et al.,1995 \\
\hline & Ramitinga & Burkina Faso & 19,30 & 0,64 & Moreau et al.,1995 \\
\hline & Bontanga reservoir & Ghana & 36,75 & 0,26 & Kwarfo-Apegyah et al., 2008 \\
\hline & Lac Mariut & Égypte & 29,80 & 0,98 & Jensen 1957 \\
\hline & Lac Tchad & Tchad & 31,40 & 0,60 & Blache 1964 \\
\hline & Lac Tchad & Tchad & 23,80 & 0,34 & Lauzanne 1978 \\
\hline \multirow{7}{*}{ C. zillii } & Samandeni & Burkina Faso & 22,87 & 0,95 & Etude en cours \\
\hline & Dangouindougou & Burkina Faso & 31,00 & 0,67 & Sirima et al., 2009 \\
\hline & Tounoura & Burkina Faso & 25,00 & 0,77 & Sirima et al., 2009 \\
\hline & Bontanga reservoir & Ghana & 21,53 & 0,60 & Kwarfo-Apegyah et al., 2008 \\
\hline & Moyen Niger & Niger & 30,96 & 0,53 & Daget 1956 \\
\hline & Lac Mariut & Égypte & 28,81 & 0,33 & Jenien 1957 \\
\hline & Etangs d'Égypte & Egypte & 37,87 & 0,19 & El Bolock et Koura, 1960 \\
\hline \multirow{3}{*}{ M. senegalensis } & Samandeni & Burkina Faso & 31,43 & 1,18 & Etude en cours \\
\hline & Okpara stream & Benin & 18,90 & 1,6 & Imorou et al., 2019 \\
\hline & Bontanga reservoir & Ghana & 24,68 & 0,69 & Kwarfo-Apegyah et al., 2008 \\
\hline \multirow{5}{*}{ S. intermedius } & Samandeni & Burkina Faso & 33,71 & 0,58 & Etude en cours \\
\hline & Cross river & Nigéria & 27,50 & 0,29 & Etim et al., 1999 \\
\hline & Okpara stream & Benin & 25,20 & 0,66 & Imorou et al., 2019 \\
\hline & Lac Nokoue & Benin & 26,00 & 0,7 & Niyonkuru, 2007 \\
\hline & Volta-Yapei, & Ghana & 27,93 & 0,95 & Abobi and Ekau, 2013 \\
\hline
\end{tabular}


Diversité piscicole et paramètres démographiques des principales espèces de p...

\section{Conclusion}

Cette étude a permis de connaitre les espèces de poisson présentes dans le réservoir de Samandeni et d'actualiser la liste la faune ichtyologique du sous bassin de Mouhoun supérieur, lieu d'implantation dudit réservoir. Certaines espèces jadis signalées ou la proportion espérée de certaines d'entre elles n'ont pas été observées au cours de cette étude. Soit, ces espèces ont été perturbées par les conditions nouvelles liées à la création du réservoir, soit elles se sont retirées dans des habitats que notre travail de prospection n'a pas permis d'atteindre. Les filets de petites mailles, notamment de $10 \mathrm{~mm}$ de côté de maille, ont rarement été utilisés au cours du présent travail. À cela s'ajoute l'inaccessibilité en saison pluvieuse des sites situés dans la partie haute du réservoir qui n'ont été prospectés que partiellement. Ainsi, des espèces, notamment celles de petites tailles ou filiformes, ont certainement échappées à ce travail d'inventaire. La construction du barrage de Samandéni convertira une partie du Fleuve Mouhoun en milieu lentique. Ce qui génére des bouleversements dans la communauté piscicole. Cependant une pêcherie florissante pourrait y être créée. Dans un tel contexte, il est essentiel de poursuivre les efforts d'étude des composantes biotique et abiotique de ce nouvel écosystème, ce qui contribuera à sa gestion durable.

\section{Remerciements}

Nous remercions le projet Susfish plus et la Direction Générale des Ressources Halieutiques du Burkina Faso pour l'assistance financière et logistique reçue lors de la collecte des données sur le site.

\section{Bibliographie}

Akombo P.M, Akange E.T Atile J.I., 2015. Age and growth of catfish Synodontis schall, (Bloch and Schneider , 1801 ) in the Lower Benue River, at. Int. J. Fish. Aquat. Stud. 2(5), 184-190.

Attakin E., 2006. Avant-projet détaillé du barrage de Samendeni. Etude d'impact sur l'environnement et le social. Mémoire de fin de formation d'études supérieures spécialisées. 2iE Groupe EIER/ETSHER, Burkina Faso.

Baijot E., Moreau J., Bouda S., 1994. Aspects hydrologiques et piscicoles des retenues d'eau en zone Soudano-sahélienne. Le cas du Burkina Faso. Cent. Tech. Coopération Agric. Rural. ACP/CEE Burkina Faso.

Bancé V., Ouéda A., Kaboré I., Zerbo H., Kabré B.G., 2021. Ecological status of a newly impounded sub-saharan reservoir based on benthic macroinvertebrates community (Burkina Faso, West Africa). Journal of Ecology and The Natural Environment, 13(1), 1-10.

Belem M, Zoungrana M, Nabaloum M., 2018. Les effets combinés du climat et des pressions anthropiques sur la forêt classée de Toéssin, province du Passoré, Burkina Faso. International Journal of Biological and Chemical Sciences, 12(5), 2186-2201.Burkina Faso., 2016. Plan National de Développement Economique et Social ( PNDES ) 2016-2020.

Cecchi P., 2009. Quelle pêche au Burkina Faso? Programme d'appui au développement l'agriculture du Burkina Faso phase 2 (PADAB 2) Compos. $n^{\circ} 2$ développement Rural décentralisée actions Pilot. Propos. en Piscic. dans les régions est/centre est/Sahel 34-47. 
Da N., Ouédraogo R., Ouéda A., 2018. Relation poids-longueur et facteur de condition de Clarias anguillaris et Sarotherodon galilaeus pêchées dans le lac Bam et le réservoir de la Kompienga au Burkina Faso. Int. J. Biol. Chem. Sci. 12(4), 1601, DOI:10.4314/ijbcs.v12i4.8.

Dakouré D., 2003. Etude hydrogéologique et géochimique de la bordure Sud-Est du bassin sédimentaire de Taoudéni (Burkina Faso - Mali) - Essai de modélisation, PhD thesis, Université Paris 6 - Pierre et Marie Curie, France.

Danadu.M.C., 2012. Problematique de Synodontis cuvier, 1816 (Siluriformes, Mochokidae) dans le bassin du fleuve Congo: systematique et écologie (R.D.C.). Thèse Dr. Univ. KISANGANI Fac. DES Sci. 2013-2014.

De Mérona B., Vigouroux R., Tejerina-Garro F.L., 2005. Alteration of fish diversity downstream from Petit-Saut Dam in French Guiana. Implication of ecological strategies of fish species. Hydrobiologia 551(1), 33-47, DOI:10.1007/s10750-005-4448-z.

Dibala et al., 2018. Production du tilapia du Nil (Oreochromis niloticus linneaus , 1758 ) avec des aliments à base de protéines végétales. Journal of Applied Biosciences,128 (2018) 12943 -12952.

Dibloni et al., 2009. Caractérisation paysanne de Hippopotamus amphibius Linné 1758, dans la Réserve de Biosphère de la Mare aux Hippopotames, en zone sud soudanienne du Burkina Faso. Int. J. Biol. Chem. Sci. 3(2), 386-397, DOI:10.4314/ijbcs.v3i2.44515.

Froese R. \& Pauly D., 1999. FishBase. World Wide Web Electron. Publ. www.fishbase.org, 2.

Gosse J.P., 1963. Le milieu aquatique et l'écologie des poissons dans la région de Yangambi. Ann. Mus. Roy. Afr. Centr., 11 113-271, 17 fig., 10 Pl.Gulland J., 1969. Manual of Methods of Fish Stock Assessment. Part L Fish Popul. Anal. FAO Man. Fish. Sci. 4, 154 pp.

Hermi M. \& Aissa P., 2002. Impact de l'anthropisation du Lac Sud de Tunis ( Tunisie ) sur la structure automnale des peuplements de nematodes libres . Structure 29, 77-85.

H Hugueny B., 1989. West African rivers as biogeographic islands: species richness of fish communities. Oecologia 79(2), 236-243, DOI:10.1007/BF00388483.

Kabré G.B., Sakiti N.G., Marques A. \& Sawadogo L., 2002. Thelohanellus bicornei n. sp. Myxosporidie (Myxosporea, Bivalvulida) parasite des branchies de Labeo coubie Rüppel, 1832 (Osteichthyen, Cyprinidae) au Burkina Faso, Afrique de l'Ouest. Parasite 9(3), 219-223, DOI:10.1051/parasite/2002093219.

Lalèyè et al., 2004. Étude de la diversité ichtyologique du bassin du fleuve ouémé au bénin (Afrique de l'ouest). Cybium 28(4), 329-339.

Lauzanne L., 1988. Les habitudes alimentaires des poissons d'eau douce africains (Feedings habits of African freshwater fishes) In. Biologie et écologie des poissons d'eau douce Africains. ORSTOM. Col.Travaux Doc. 216 221- 242.

Leveque C., \& Paugy D., 1999. Les poissons des eaux continentales africains. Diversité, écologie, utilisation par l'homme. Paris, IRD, 521 p.Mano et al., 2019. Fish assemblages in the Upper part of the Volta River, Burkina Faso: A link analysis towards fisheries management and conservation. Int. J. Biol. Chem. Sci. 13(6), 2546, DOI:10.4314/ijbcs.v13i6.11. 
Diversité piscicole et paramètres démographiques des principales espèces de p...

Melcher A.H., Ouedraogo R. \& Schmutz S., 2012. Spatial and seasonal fish community patterns in impacted and protected semi-arid rivers of Burkina Faso 48, 117-129, DOI:10.1016/ j.ecoleng.2011.07.012.

Minoungou M., Ouédraogo R., Da N., Oueda A., 2020. Relation longueur-poids et facteur de condition de sept espèces de poisson du réservoir de Samandeni avant son ouverture à la pêche (Burkina Faso). J. Appl. Biosci. 151(April 2018), 15559-15572, DOI:10.35759/jabs.151.5.

Ouéda A., Guenda W., Ouattara A., Gourème G., Hugueny B., Kabre G.B., 2008. Seasonal diet of shift of the most important fish species in a sahelo-soudanian reservoir (Burkina Faso). J. Fish. Aquat. Sci. 3 240-251.

Ouédraogo R., 2010. Fish and fisheries prospective in arid inland waters of Burkina Faso, West Africa. ONORM, 2010. M 6232, QZV Okologie OG BG. Bl. II Nr. 99/2010. PhD Thesis, University of Natural Resources and Life Sciences, Vienna. (November), 232.

Ouédraogo R., Aïcha S. \& Adama O., 2015. Description du peuplement piscicole du lac sahélien de Higa , un site Ramsar du Burkina Faso , Afrique de l’Ouest (May 2012), 8958-8965.

Paugy D. \& Bénech V., 1989. Les poissons des bassins d'eau douce des bassins côtiers du Togo (Afrique de l'Ouest). Conv. OMS-ORSTOM 23 l-110.Paugy D., Lévêque C., Teugels G.G., 2003. Poissons d'eaux douces et saumâtres de l'Afrique de l'Ouest, édition complète. Tome I \& II (Vol. 815). Edition IRD-MNHN-MRAC, Paris-Turvuren. 457.

Pauly D., 1997. Méthode pour l'évaluation des ressources halieutiques.(adaptation française: J. Moreau). Cépaduès éditions, Toulouse, Fr. 288pp.

Pauly D. 1980. On the interrelationships between natural mortality, growth parameters, and mean environmental temperature in 175 fish stocks. ICES journal of Marine Science, 39(2), 175-192.

Pauly D., 1985. Population dynamics of short-lived species, with emphasis on squids. NAFO Sci. Coun. Stud. 9(207), 143-154.PHIE, 2019. Rapport d'activités annuel 2019 du perimètre halieutique d'intérêt éconimique de Samandeni.

Pinton A., 2008. Anatomie, systématique et phylogénie de poissons Synodontis actuels et fossiles (Siluriformes, Mochokidae): implications dans la paléobiogéographie intracontinentale néogène d'Afrique. (Doctoral Diss. Poitiers).

Poda J.N., Traoré A. \& Sondo B.K., 2004. L’endémie bilharzienne au Burkina Faso. Bull. la Soc. Pathol. Exot. 97(1), 29-32.

Présidence du Faso., 2014. DECRET N 2014-791/PRES/PM/MRAH/MEF du 16 septembre 2014 portant adoption de la Stratégie Nationale de Développement Durable de la Pêche et de l’Aquaculture au Burkina Faso (SN-DDPA). JO N 472014.

Roman B., 1966. Les Poissons des Hauts- Bassins de la Volta. Annales - Série IN-8 - Sciences Zoologiques - N 150. Musée Royale de l’Afrique Centrale; Tervuren, Belgique. 191 p+ planche.

Sirima O., Toguyeni A. \& Kabore-Zoungrana C., 2009. Faune piscicole du bassin de la Comoé et paramètres de croissance de quelques espèces d'intérêt économique. Int. J. Biol. Chem. Sci. 3(1), 95-106, DOI:10.4314/ijbcs.v3i1.42740. 
Toguyeni et al., 1996. Consequences of food restriction on short-term growth variation and on plasma circulating hormones in Oreochromis niloticus in relation to sex. Gen. Comp. Endocrinol. 103(2), 167-175, DOI:10.1006/gcen.1996.0107.

Traoré R., 2012. Eau, territoire et conflits : analyse des enjeux de la gestion communautaire de l'eau au Burkina Faso : l'exemple du bassin versant du Nakambé. (Doctoral dissertation, Université Toulouse le Mirail-Toulouse II).

Von Bertalanffy L., 1938. A quantitative theory of organic growth (inquiries on growth laws. II). Hum. Biol. 10(2), 181.

Yonkeu S., Traore O., Yomba Keptukwa S., 2009. Étude d’impact environnemental et social de la mise en place du barrage à buts multiples de Samandeni au Burkina Faso: volet centrale hydroélectrique. Liaison énergie francophonie, (83), 32-41.

Zampaligre I., 2004. Etude de l'effort de pêche et proposition de scenario de régulation sur les pêcheries de Kompienga et Bagré. Élaboration de protocole expérimental pour la mise au point de systèmes sélectifs de pêches d’espèces de petites tailles. Rapport final. Direction. Juin 2004. 52 p.

PDF généré automatiquement le 2023-04-26 15:27:27

Url de l'article : https://popups.uliege.be/2295-8010/index.php?id=1948 